\title{
TURBULENT KINETIC ENERGY BUDGETS FROM A LARGE-EDDY SIMULATION OF AIRFLOW ABOVE AND WITHIN A FOREST CANOPY
}

\author{
MICHAEL J. DWYER, * EDWARD G. PATTON and ROGER H. SHAW \\ Department of Land, Air and Water Resources, University of California, Davis, CA 95616, U.S.A.
}

(Received in final form 21 February, 1997)

\begin{abstract}
The output of a large-eddy simulation was used to study the terms of the turbulent kinetic energy (TKE) budget for the air layers above and within a forest. The computation created a threedimensional, time-dependent simulation of the airflow, in which the lowest third of the domain was occupied by drag elements and heat sources to represent the forest. Shear production was a principal source of TKE in the upper canopy, diminishing gradually above tree-top height and more sharply with depth in the canopy. The transfer of energy to subgrid scales (dissipation) was the main sink in the upper part of the domain but diminished rapidly with depth in the canopy. Removal of resolvedscale TKE due to canopy drag was extremely important, occurring primarily in the upper half of the forest where the foliage density was large. Turbulent transport showed a loss at the canopy top and a gain within the canopy. These general features have been found elsewhere but uncertainty remains concerning the effects of pressure transport. In the present work, pressure was calculated directly, allowing us to compute the pressure diffusion term. Well above the canopy, pressure transport was smaller than, and opposite in sign to, the turbulent transport term. Near the canopy top and below, pressure transport acted in concert with turbulent transport to export TKE from the region immediately above and within the upper crown, and to provide turbulent energy for the lower parts of the forest. In combination, the transport terms accounted for over half of the TKE loss near the canopy top, and in the lowest two-thirds of the canopy the transport terms were the dominant source terms in the budget. Moreover, the pressure transport was the largest source of turbulent kinetic energy in the lowest levels of the canopy, being particularly strong under convective conditions. These results indicate that pressure transport is important in the plant canopy turbulent kinetic energy budget, especially in the lowest portion of the stand, where it acts as the major driving force for turbulent motions.
\end{abstract}

Key words: Turbulent kinetic energy, Large-eddy simulation, Canopy

\section{Introduction}

Turbulent kinetic energy (TKE) budgets illustrate the relative importance of physical processes that govern turbulent fluid motions. The presence of a plant canopy modifies the surface boundary layer in a unique fashion and adds further dimension to the balance of TKE. Most notably, the canopy imposes aerodynamic drag on the flow and creates turbulent motions in the wakes of the plant elements. This latter process is an additional source of TKE (wake production) as kinetic energy passes from the mean flow to turbulence (Wilson and Shaw, 1977; Raupach and Shaw, 1982; Raupach et al., 1986), and also represents a transfer of turbulent kinetic energy from 'large' scales to wake scales (Shaw and Seginer, 1985). In both aspects, consideration must be given to the scale of motion generated in such wakes. In

* Current Affiliation: Air Force Weather Center, Offutt A.F.B., NE 68113, U.S.A. 
addition, the canopy may act as a heat source or sink, or both, depending on the temperature difference between layers of the canopy and the surrounding air at each level.

Lesnik (1974) provided one of the earliest pictures of the canopy TKE budget, showing data from a twenty year old pine forest under different stability conditions. Shear production was a major source at the canopy top where the wind shear gradient was largest, and dissipation was a large loss in the upper canopy. Buoyancy was small in comparison with the other terms and was not shown. The unique feature of the budget presented by Lesnik was the role of the turbulent transport term, in which a significant gain in the lower canopy levels represented an import of turbulent energy from the primary production region in the upper canopy levels.

More recent field experiments support these results. For example, Meyers and Baldocchi (1991) present TKE budgets for a deciduous forest under neutral stability, in which profiles of shear production, turbulent transport and dissipation (the latter found as a residual) were in qualitative agreement with Lesnik (1974). Meyers and Baldocchi (1991) estimated wake production and found it to slightly exceed shear production at all levels within the canopy except near the canopy top.

The effects on the TKE budget of atmospheric thermal stratification were investigated by Leclerc et al. (1990) using data from a deciduous forest collected during the Camp Borden experiment (Shaw et al., 1988). In general, their measurements showed that normalized shear production and dissipation, found as a residual, increase with decreasing levels of convective instability, and increase sharply again with the onset of stable conditions.

Some have considered it appropriate to present the budget with TKE split into two different scales, turbulent shear kinetic energy (SKE) and turbulent wake kinetic energy (Shaw and Seginer, 1985; Wilson, 1988). Presentation of the budget in this manner supposes that the canopy acts to create a loss of kinetic energy for the 'large' shear induced scales, and a gain for the 'small' wake scales. This process represents the action of the drag elements to suppress turbulent motions as well as to suppress the mean flow and, at the same time, create small-scale motions in the wake of plant elements. Presenting the budget in this manner, Leclerc et al. (1990) showed that canopy drag was the primary destruction term in the SKE budget, while shear production was the primary source in the upper canopy. Turbulent transport was a loss near the canopy top and a gain within the canopy. Under unstable conditions, the residual term, representing the sum of viscous dissipation, pressure transport and accumulated errors, was a gain within the canopy and a loss at the canopy top and above. Since dissipation can only act to eliminate TKE, this is evidence that pressure transport is a source within the canopy.

Such field experiments contribute to our understanding of turbulent flow within a canopy. However, the effects of turbulent pressure fluctuations are still largely uncertain. Because of characteristically high turbulence intensities, direct measurements of turbulent pressure fluctuations in plant canopies are extremely difficult but there are examples of such measurements (Sigmon et al., 1983). Measurements 
at the soil surface are less troublesome and have been presented by Maitani and Seo (1985), Shaw et al. (1990), and Shaw and Zhang (1992).

Maitani and Seo (1985) measured surface pressure fluctuations in wheat, and vertical velocity within and above the canopy. They assumed that pressure fluctuations at the levels of the anemometers were approximated by the surface measurements, from which calculations of pressure-velocity covariances indicated that the pressure driven flux of TKE was downward, and that the pressure transport term in the TKE budget was not negligibly small. Shaw et al. (1990) and Shaw and Zhang (1992) used surface pressure and tower-based velocity measurements from the Camp Borden forest study to infer the role of pressure fluctuations in canopy turbulence. Shaw et al. (1990) compared surface pressure measurements to pressure fluctuations calculated through a Poisson equation to demonstrate that pressure fluctuations at the surface are primarily created by velocity perturbations in the high shear region near the top of the forest. Shaw and Zhang (1992) found that longitudinal velocities measured in the trunk space were strongly correlated with surface pressure, and that peak correlations occurred with near-zero time lag. They proposed this as evidence that turbulence at low levels in the forest is to a large extent driven by pressure fluctuations. Pressure induced motions in the lower canopy are consistent with a significant pressure transport term in the TKE budget.

Zhuang and Amiro (1994) calculated the two-dimensional field of perturbation pressure from a Poisson equation using the Camp Borden data. In terms of pressure diffusion of TKE, however, their results are inconclusive, since they did not separate pressure diffusion and return-to-isotropy components of the pressure-velocity interactions. In addition, their analysis was necessarily confined to periods identified as coherent structures because they needed to apply Taylor's hypothesis to convert from temporal to spatial rates of change. Another limitation arises from their need to neglect contributions from lateral velocity gradients.

An alternative to field experiments has been the use of closure models to investigate canopy budgets (Wilson and Shaw, 1977; Meyers and Paw U, 1986; Wilson, 1988; Meyers and Baldocchi, 1991). Although these models provide qualitative agreement with field measurements or estimates of shear production, wake production, dissipation, and turbulent transport, pressure effects are either assumed negligible or parameterized. As a result, no conclusions can be drawn regarding the role of pressure perturbations in the budgets.

Others have turned to wind-tunnel studies to investigate the flow of air within and above a plant canopy. Most notable are the studies of Raupach et al. (1986) who used a model canopy constructed of aluminum strips, and Brunet et al. (1994) who simulated airflow through a wheat canopy using flexible nylon stalks. Budgets were presented that are in qualitative agreement with field experiments and mathematical models. Moreover, both studies offer insight into the possible role of the turbulent pressure perturbations within and above a plant canopy. In the TKE budget, Raupach et al. (1986) found significant differences between two calculations of the dissipation. They suggested that the difference may be due to pressure 
transport indicating that pressure diffusion is a source above the canopy in approximate balance with turbulent transport, and that pressure and turbulent transport are both sources of TKE within the canopy. Brunet et al. (1994) also suggested that pressure transport is a source above the canopy. However, their residual analysis indicated that pressure transport is a loss within the canopy, which contradicts the earlier results of Raupach et al. (1986). Differences between the two wind-tunnel studies appear to arise from substantial differences in estimates of viscous dissipation within the canopy. Brunet et al. (1994) stated that, 'there is clearly an urgent need for high-quality measurements of fluctuating pressure in the context of plant canopy flows'.

While output from a large-eddy simulation (LES) should not be considered an adequate substitute for accurate field measurements, it is likely that LES can provide evidence concerning the role of pressure perturbations in the dynamics of canopy air flow. Shaw and Schumann (1992) were the first to employ the LES technique to the plant canopy environment. Simulated mean velocity profiles, vertical profiles of Reynolds stress, turbulent kinetic energy, and velocity skewness provided qualitative agreement with observations. Kanda and Hino (1994) also used LES to explore coherent motions within and above a plant canopy, but their simulation was limited to the developing stage of turbulence and not to a fully developed flow regime.

In the present study, the code of Moeng (1984) and Moeng and Wyngaard (1988), modified by Patton et al. (1994) to include a plant canopy, is used to simulate canopy flow fields. While it has been useful for us to consider our simulations as applying to the flow field through and above a forest, the equations and the representation of the canopy (leaf area distribution and drag coefficient) could relate equally well to a stand of quite different height. The motivation is to use the three-dimensional output of velocity, temperature, subgrid-scale kinetic energy, and pressure to calculate all terms in the resolved-scale turbulent kinetic energy budget with particular emphasis on determining the role of the pressure perturbations in the budget. Several simulations have been completed with different canopy and environmental specifications, and comparisons are made between simulations and with field experiments, closure models, and wind-tunnel results.

\section{Methods}

\subsection{EQUATIONS}

Large-eddy simulation explicitly calculates the large or resolved-scales of turbulent flow while the small scales, the subgrid-scales, are parameterized. The assumption is made that the resolved-scales contain most of the energy and are fairly insensitive to the effects of the subgrid-scale parameterizations. While this is adequately demonstrated for planetary boundary-layer scale simulations (Nieuwstadt et al., 
1993; Andren et al., 1994), it remains to be sufficiently justified with regard to canopy flow. It is encouraging, however, that Kaimal and Finnigan (1994), summarizing numerous reports of canopy wind spectra, state that spectral peaks do not vary with height on descent 'through the roughness sublayer to mid-canopy height', such that the canopy layers are not dominated by small-scale turbulence.

The resolved-scale is defined using a wave-cut-off filter in the horizontal, and a grid-volume average in the vertical (Moeng and Wyngaard, 1988). The derivation of the resolved-scale equations can be found in Moeng (1984) but some details will be presented here for completeness and to show the terms that represent the presence of the plant canopy. Specifically, a form drag term and a canopy heat source are added to the resolved-scale momentum and thermal energy equations respectively.

Under the Boussinesq approximation, the resolved-scale conservation equations for mass, momentum and energy are written in a form consistent with that of Moeng (1984),

$$
\begin{aligned}
& \frac{\partial \bar{u}_{i}}{\partial x_{i}}=0 \\
& \frac{\partial \bar{u}_{i}}{\partial t}=-\bar{u}_{j}\left(\frac{\partial \bar{u}_{i}}{\partial x_{j}}-\frac{\partial \bar{u}_{j}}{\partial x_{i}}\right)-\frac{\partial \bar{\pi}}{\partial x_{i}}-\frac{1}{\rho_{\circ}} \frac{\partial\langle\bar{p}\rangle}{\partial x_{i}} \delta_{i 1}+\frac{g}{\theta_{\circ}} \bar{\theta} \delta_{i 3}-\frac{\partial \tau_{i j}}{\partial x_{j}}+F_{i} \\
& \frac{\partial \bar{\theta}}{\partial t}=-\bar{u}_{j} \frac{\partial \bar{\theta}}{\partial x_{j}}-\frac{\partial \tau_{\theta j}}{\partial x_{j}}+S .
\end{aligned}
$$

The filter separates flow variables into resolved-scale and subgrid-scale (SGS) components, such that velocity $u_{i}=\bar{u}_{i}+u_{i}^{\prime} \cdot \bar{\theta}$ is potential temperature, $1 / \theta_{\circ}$ is the volumetric expansion coefficient, $g$ is gravitational acceleration, $\rho_{\circ}$ is density, $\bar{p}$ is pressure, $\tau_{i j}$ is the SGS shear stress, and $\tau_{\theta j}$ is the SGS heat flux. The canopy effects of form drag and heat source are represented as $F_{i}$ and $S$, respectively.

The SGS terms arise from application of the wave-cut-off filter (Moeng and Wyngaard, 1988). In the momentum equation, we follow Moeng (1984) and write the subgrid-scale stress tensor as

$$
\tau_{i j}=R_{i j}-R_{k k} \delta_{i j} / 3,
$$

where

$$
R_{i j}=\overline{u_{i}^{\prime} u_{j}{ }^{\prime}} .
$$

The normal components have been subtracted from the stress tensor and added as SGS dynamic pressure to the static pressure to form the quantity

$$
\bar{\pi}=\frac{\bar{p}^{\prime \prime}}{\rho_{\circ}}+\frac{R_{k k}}{3}+\frac{\bar{u}_{j} \bar{u}_{j}}{2},
$$


where $\bar{p}^{\prime \prime}$ is the deviation of the resolved pressure from its horizontal mean $\langle\bar{p}\rangle$, since the mean pressure gradient is shown separately in Equation (2) as an external forcing in the $x_{1}$-direction. The third term on the right hand side of Equation (6) is the resolved-scale dynamic pressure which arises when the advective term is expressed in rotational form as in Equation (2). The substitution of Equation (6) into the resolved-scale momentum equation yields

$$
\begin{aligned}
\frac{\partial \bar{u}_{i}}{\partial t}= & -\bar{u}_{j}\left(\frac{\partial \bar{u}_{i}}{\partial x_{j}}-\frac{\partial \bar{u}_{j}}{\partial x_{i}}\right)-\frac{1}{2} \frac{\partial \bar{u}_{j} \bar{u}_{j}}{\partial x_{i}}-\frac{2}{3} \frac{\partial \overline{\bar{e}^{\prime}}}{\partial x_{i}} \\
& -\frac{1}{\rho_{\circ}} \frac{\partial \bar{p}^{\prime \prime}}{\partial x_{i}}-\frac{1}{\rho_{\circ}} \frac{\partial\langle\bar{p}\rangle}{\partial x_{i}} \delta_{i 1}+\frac{g}{\theta_{\circ}} \bar{\theta} \delta_{i 3}-\frac{\partial \tau_{i j}}{\partial x_{j}}+F_{i},
\end{aligned}
$$

where $\overline{e^{\prime}} \equiv R_{k k} / 2$ is SGS kinetic energy.

At each node, a time-dependent drag force was parameterized in a conventional manner as the product of a drag coefficient, an element area density, and the square of the velocity. The drag force in the $x_{i}$-direction is

$$
F_{i}=-C_{d} a(z) V \bar{u}_{i},
$$

where $V$ is the instantaneous scalar wind speed, $C_{d}$ is an isotropic drag coefficient assumed constant and equal to 0.15 according to measurements in a deciduous forest (Shaw et al., 1988), and $a(z)$ is plant area density.

Since no attempt was made to simulate the thermal energy budget of the canopy, we followed the procedure of Shaw and Schumann (1992), whereby the magnitude of the heat source was greatest in the upper levels where the solar radiation load would be strongest, and weakest in the lower canopy where solar radiation is attenuated. form

The subgrid-scale kinetic energy was evaluated using a budget equation of the

$$
\begin{aligned}
\frac{\partial \overline{e^{\prime}}}{\partial t}= & -\overline{u_{i} \frac{\partial \overline{\bar{e}^{\prime}}}{\partial x_{i}}}-\overline{u_{i}^{\prime} u_{j}^{\prime}} \frac{\partial \bar{u}_{i}}{\partial x_{j}}+\frac{g}{\theta_{\circ}} \overline{u_{i}^{\prime} \theta^{\prime}} \delta_{i 3} \\
& -\frac{\partial\left[\overline{u_{i}^{\prime}\left(e^{\prime}+p^{\prime} / \rho_{\circ}\right)}\right]}{\partial x_{i}}-\epsilon-2 \frac{\overline{e^{\prime}}}{\tau},
\end{aligned}
$$

where the terms on the right hand side represent, in order, advection of SGS kinetic energy by the resolved-scale velocity, shear production due to the action of SGS Reynolds stresses on the resolved-scale velocity gradient, SGS buoyancy production, SGS transport, and viscous dissipation, while the final term represents an enhancement to the dissipation rate due to the presence of the plant canopy. Here, $\tau$ is a time constant for the drag process and is equated to the resolved-scale 
velocity divided by the drag force. Shaw and Schumann (1992) also added this last term to represent the removal of SGS kinetic energy by the action of canopy drag, based on the assumption that eddies created in the wakes of the plant elements are smaller again than the most energetic SGS eddies, dissipate rapidly and make no significant contribution to SGS kinetic energy.

To solve Equation (9), Kolmogorov's hypothesis was applied for the dissipation rate, and gradient diffusion approximations were applied to the SGS fluxes. The SGS fluxes of momentum and heat also appear in the equations for resolved-scale quantities. Specifically, we expressed them in the following manner

$$
\begin{aligned}
\tau_{i j} & =-K_{M}\left(\frac{\partial \bar{u}_{i}}{\partial x_{j}}+\frac{\partial \bar{u}_{j}}{\partial x_{i}}\right), \\
\tau_{\theta j} & =-K_{H} \frac{\partial \bar{\theta}}{\partial x_{j}},
\end{aligned}
$$

where the SGS eddy diffusivities, $K_{M}$ and $K_{H}$, were assumed functions of the subgrid-scale kinetic energy and a dissipation length. Further details of the subgridscale energy budget and fluxes may be found in Moeng (1984).

\subsection{NUMERICAL MODEL}

Our computational domain was 96 x 96 nodes in the horizontal and 31 nodes in the vertical. With a grid spacing of 2 metres equidistant in all directions, this represented a domain size of $192 \mathrm{~m} \times 192 \mathrm{~m} \times 62 \mathrm{~m}$, with the lowest one-third (10 grid intervals) occupied by a $20 \mathrm{~m}$ tall forest. The governing equations were integrated in time using a second-order Adams-Bashforth scheme, with a time step of $0.1 \mathrm{~s}$. The horizontal derivatives were evaluated using a pseudospectral method (Fox and Orzag, 1973), and the vertical derivatives employed secondorder centered finite differences. The boundary conditions in the horizontal were periodic, the upper boundary was specified as a frictionless rigid lid with zero mass, momentum, heat and SGS kinetic energy flux, and the bottom boundary employed a no-slip condition with a prescribed roughness length, $z_{\circ} / h=0.001$.

The forest was defined as a vertical distribution of leaf area density. Figure 1 shows the two artificial profiles used in this study for a sparse canopy, leaf area index $(L A I)$ equal to 2 , and a dense canopy with $L A I=5$.

The conservation equations were solved on a non-staggered horizontal grid and a staggered vertical grid. The vertical velocity, $\bar{w}$, the subgrid-scale kinetic energy, $\overline{e^{\prime}}$, and the leaf area density were defined at the surface and on equally spaced grids above. The streamwise velocity, $\bar{u}$, the lateral velocity, $\bar{v}$, potential temperature, $\bar{\theta}$, and the quantity $\bar{\pi}$, were defined at a half grid point above the surface and at all intermediate levels above. The budget terms were calculated at these intermediate levels, resulting in the budget terms being offset from the location of the leaf area density. 


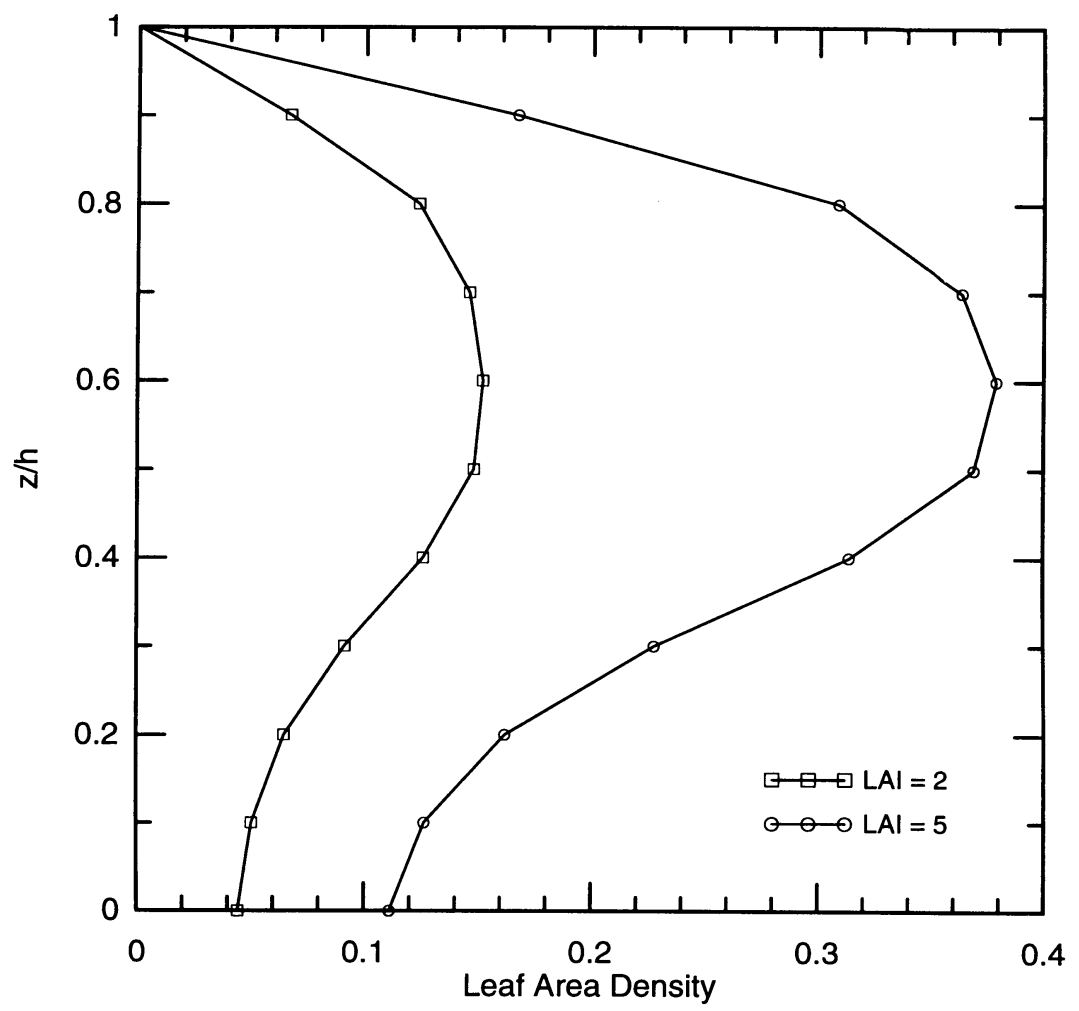

Figure 1. Vertical distribution of leaf area density $\left(\mathrm{m}^{-1}\right)$. Integration gives leaf area indices $(L A I)$ of 2 and 5.

Table I

Plant canopy variables and forcing

\begin{tabular}{llllll}
\hline Simulation & $L A I$ & $\begin{array}{l}Q_{*} \\
\left(\mathrm{~m} \mathrm{~s}^{-1} \mathrm{~K}\right)\end{array}$ & $\begin{array}{l}U \\
\left(\mathrm{~m} \mathrm{~s}^{-1}\right)\end{array}$ & $h / L$ & $\begin{array}{l}u_{*} \\
\left(\mathrm{~m} \mathrm{~s}^{-1}\right)\end{array}$ \\
\hline S1 & 2.0 & 0.005 & 2.0 & -0.024 & 0.388 \\
S5 & 5.0 & 0.005 & 2.0 & -0.017 & 0.424 \\
S6 & 5.0 & 0.125 & 4.0 & -0.064 & 0.801 \\
S7 & 5.0 & 0.005 & 1.0 & -0.153 & 0.209 \\
S8 & 5.0 & 0.125 & 1.0 & -1.252 & 0.302 \\
\hline
\end{tabular}

$L A I=$ leaf area index; $Q_{*}=$ canopy top heat flux; $U=$ mean wind speed; $h=$ canopy height; $L=$ Obukhov length; $u_{*}=$ canopy top friction velocity

The flow was externally forced by a horizontal mean pressure gradient. This pressure gradient was adjusted to ensure a constant integrated mass flux across the upwind $y, z$ boundary, which we express in terms of an area averaged wind speed, 
$U$. The wind speed was varied between simulations in order to study environmental influences on the TKE budget. The canopy density and the strength of the heat flux were also varied between simulations to determine canopy influences. Table I summarizes environmental and canopy conditions. The simulations are ordered in terms of the stability parameter $h / L$ (Shaw et al., 1988), where $h$ is the height of the canopy and $L$ is the Obukhov length evaluated at the canopy top.

After each simulation reached equilibrium, data sets were saved at specified intervals ( 250 or 500 timesteps). A data set contains the three-dimensional output of the resolved-scale velocity $(\bar{u}, \bar{v}, \bar{w})$, the resolved-scale potential temperature $(\bar{\theta})$, the SGS kinetic energy $\left(\overline{e^{\prime}}\right)$, and the quantity $\bar{\pi}$. However, since $\bar{\pi}$ contains dynamic pressure components, it was necessary to recalculate the static pressure perturbation field $\left(\bar{p}^{\prime \prime} / \rho_{\circ}\right)$. At this point all terms of the resolved-scale TKE budget can be calculated.

\subsection{The TKE BUdGET EQUATION}

The resolved-scale TKE budget written in mixed notation for steady state, horizontally homogeneous conditions is

$$
\begin{aligned}
\frac{\partial\left\langle\bar{E}^{\prime \prime}\right\rangle}{\partial t}=0= & -\underbrace{\left\langle\bar{u}^{\prime \prime} \bar{w}^{\prime \prime}\right\rangle \frac{\partial\langle\bar{u}\rangle}{\partial z}}_{P_{s}}+\underbrace{\frac{g}{\theta_{0}}\left\langle\bar{w}^{\prime \prime} \bar{\theta}^{\prime \prime}\right\rangle}_{P_{b}}-\underbrace{\left\langle\frac{\partial \bar{w}^{\prime \prime} \bar{E}^{\prime \prime}}{\partial z}\right\rangle}_{T_{t}} \\
& -\underbrace{\left\langle\frac{1}{\rho_{0}} \frac{\partial \bar{w}^{\prime \prime} \bar{p}^{\prime \prime}}{\partial z}\right\rangle}_{T_{p}}+\underbrace{\left\langle\bar{u}_{i}^{\prime \prime} F_{i}^{\prime \prime}\right\rangle}_{D_{c d}} \\
& -\underbrace{\left\langle\frac{2}{3} \frac{\partial \bar{u}_{i}^{\prime \prime}\left(\overline{e^{\prime}}\right)^{\prime \prime}}{\partial x_{i}}+\frac{\partial \bar{u}_{i}^{\prime \prime} \tau_{i j}^{\prime \prime}}{\partial x_{j}}\right\rangle}_{T_{s g s}}+\underbrace{\left\langle\tau_{i j}^{\prime \prime} \frac{\partial \bar{u}_{i}^{\prime \prime}}{\partial x_{j}}\right\rangle}_{D_{s g s}},
\end{aligned}
$$

where $\left\langle\bar{E}^{\prime \prime}\right\rangle \equiv\left\langle\bar{u}_{i}^{\prime \prime} \bar{u}_{i}^{\prime \prime} / 2\right\rangle$ is the horizontal averaged resolved-scale turbulent kinetic energy. $P_{s}$ is shear production representing the conversion of mean flow kinetic energy to resolved-scale TKE, $P_{b}$ acts as either buoyant production or destruction depending on the sign of the vertical heat flux, $T_{t}$ is the net result of resolved-scale turbulent transport, $T_{p}$ represents the transport of resolved-scale kinetic energy by pressure fluctuations, and $D_{c d}$ represents the rate of work performed by the velocity perturbations against the drag caused by canopy elements. The two parts of $T_{\text {sgs }}$ represent subgrid-scale diffusion of resolved-scale TKE, while $D_{s g s}$ represents transfer of resolved-scale TKE to the subgrid-scales. Subgrid-scale diffusion $\left(T_{s g s}\right)$ is added to the resolved-scale turbulent transport $\left(T_{t}\right)$, and the total referred to as turbulent transport $(T T)$. The rate of transfer of resolved-scale TKE to the subgrid- 


\section{Normalized TKE Budget}

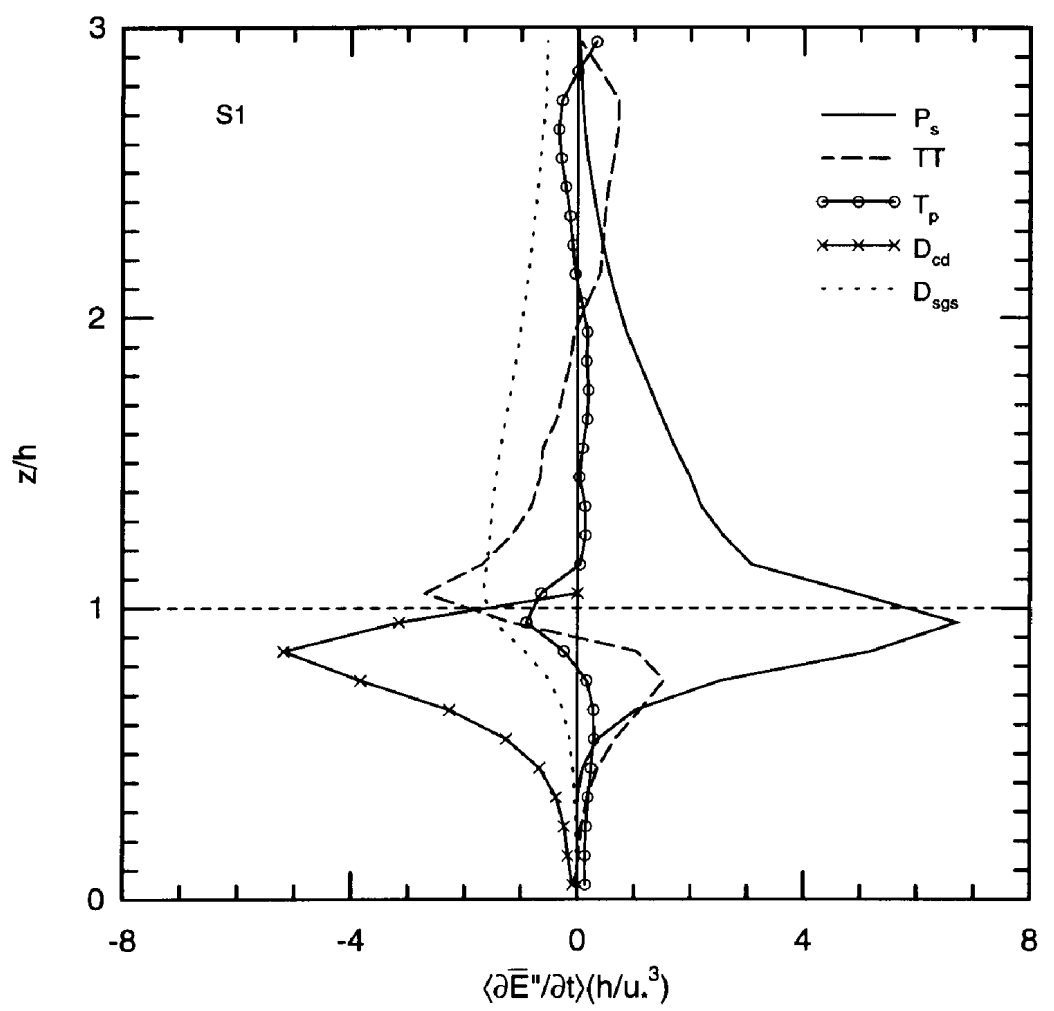

Figure 2. Normalized resolved-scale TKE budget for simulation $\mathrm{S} 1, h / L=-0.024$. Plot labels refer to budget terms in Equation (12), where $T T$ refers to the sum of $T_{t}$ and $T_{s g s}$.

scale $\left(D_{\text {sgs }}\right)$ is equivalent to a conversion of energy to the dissipation scales (Moeng, 1984; Mason, 1994).

The budgets presented here differ in certain aspects from those typically found for the canopy layer (Wilson and Shaw, 1977; Raupach and Shaw, 1982; Raupach et al., 1986). First, there is no wake production term because, with a grid spacing of $2 \mathrm{~m}$, only eddies of wavelength greater than $4 \mathrm{~m}$ are resolved. Eddies of this size are significantly larger than those expected of the wake production process, which are determined by the dimensions of the plant elements. Secondly, there are no dispersive fluxes to represent spatial correlations between time averaged quantities (Raupach and Shaw, 1982; Raupach et al., 1986). Such fluxes are absent from the equations as a consequence of formulating spatial averages of the computed flow fields. However, an ensemble average of each term in the budget equation was formed by averaging the terms over a minimum of 12 data sets within each simulation. 


\section{Results and Discussion}

As a first example, the turbulent kinetic energy budget for the sparse canopy under near-neutral conditions is shown in Figure 2, with terms normalized by height $h$ and friction velocity $u_{*}$, which is defined at $z=h$. In this low heat flux case, buoyancy is unimportant at all levels and is omitted for clarity. As in all of the cases we examined, balance was achieved in our calculations to within a few percent of the largest term at each level, suggesting that numerical errors in our budget calculations are small.

The overall features of this budget are in qualitative agreement with previous studies. The dominant production mechanism is shear, term $P_{s}$ in Equation (12), which achieves maximum strength just below tree-top height, coincident with the level of highest mean wind shear. Shear production diminishes rapidly with depth in the canopy, however, and is negligible below about $z=0.5 h$. The peak value is, in this case, equal to $6.7 u_{*}^{3} / h$, which is intermediate between normalized values of 5.9 for $\mathrm{S} 8(L A I=5, h / L=1.25)$, and 8.2 for $\mathrm{S} 5(L A I=5, h / L=0.02)$. These values compare favorably with values calculated from forest measurements. For example, Leclerc et al. (1990) record a peak normalized value of shear production of about 6 under near-neutral conditions, while Zhuang and Amiro (1994) show a peak of about 6.3, their calculations relating to conditionally sampled periods of coherent structure. On the other hand, wind-tunnel studies (Raupach et al., 1986; Brunet et al., 1994) have produced smaller values, of the order of 4 , and the deciduous forest study of Meyers and Baldocchi (1991) shows a value as high as 20. The small wind-tunnel value is probably a consequence of the low area indices of the artificial canopy elements, while this high forest value is likely to be the result of the high, dense crown of the forest in question.

The restrictive upper boundary conditions, which prevent the appearance of the constant stress and flux layer normally found in the atmospheric surface layer, force the shear production term to zero at the top of the domain. This undoubtedly has an impact on the appearance of all of the TKE budget terms, especially above $z / h$ $=2$. However, the primary source region for generation of TKE is the high shear layer near the canopy top, and this is likely to have a dominating influence on the budgets, leading us to believe that our results are realistic.

Well above the canopy, production is largely balanced by transfer to subgridscales (term $D_{s g s}$ ), which must be considered a dissipation process as far as the resolved-scale motions are concerned. Transfer to subgrid-scales is important down to about the mid-point in the canopy. Within the canopy, drag (term $D_{c d}$ ) is by far the most important term to counterbalance production. That is, canopy elements have a strong influence in suppressing resolved-scale turbulent motions, as well as reducing the mean flow. Many analyses have discounted this process because the bluff object component of the aerodynamic drag represents a conversion of energy from 'large' scales to wake scales and is not a loss of kinetic energy per se. Here we treat wake eddies as subgrid-scale, and the process of canopy drag is considered a 
'short circuit' to the traditionally recognized inertial cascade process. We contrast our interpretation with that of Brunet et al. (1994), who do not consider canopy drag in their TKE budget, even though they recognize wake turbulence as being unresolved by their measurement system.

In addition, it is clear that the diffusion terms are of crucial importance to the TKE budget; the sum of turbulent and pressure diffusion accounting for more than $50 \%$ of the TKE loss immediately above the canopy, and being responsible for nearly all turbulence in the lower half of the canopy. The combined resolvedscale and subgrid-scale turbulent transport term $(T T)$ is responsible for removing turbulent energy from the canopy top region and from a layer up to twice tree-top height, and to redistribute this energy to the upper reaches of the domain and to layers within the depth of the forest. Just above the top of the canopy, it is the largest sink for kinetic energy. As a source to the canopy, it peaks at about $0.75 h$, and then diminishes asymptotically to become insignificant below about $0.25 h$. This pattern is well supported by the results of observations in forests (Lesnik, 1974; Leclerc et al., 1990; Meyers and Baldocchi, 1991), by those of wind-tunnel experiments (Raupach et al., 1986; Brunet et al., 1994), and by higher order closure models (Wilson and Shaw, 1977; Meyers and Paw U, 1986; Meyers and Paw U, 1987; Meyers and Baldocchi, 1991).

In our simulations, subgrid-scale diffusion of resolved-scale turbulent energy is not insignificant but is generally small. Figure 3 focuses on turbulent transport $(T T)$ in the bottom half of the domain and separates $T T$ into its resolved-scale component $\left(T_{t}\right)$, and the two SGS components $\left(T_{s g s}\right)$ that are the second-from-last and third-from-last terms in Equation (12). Where TT peaks on the negative and positive sides, the combined SGS diffusion terms account for $14 \%$ and $16 \%$ of the total transport respectively.

Our calculations of the pressure diffusion term $\left(T_{p}\right)$ are of prime interest, since direct field measurements are extremely difficult, and, while previous studies have shown general agreement with regard to the turbulent transport term, this is definitely not the case for pressure diffusion. With respect to canopy and near-canopy levels, Figure 2 shows that pressure diffusion operates in a similar fashion to turbulent diffusion, extracting energy from near the canopy top, and acting as an energy supply for lower regions. The maximum size of the pressure term is smaller, however, peaking on the negative side near $z=h$ at slightly less than half that of $T T$. One major difference between the two diffusion processes is that pressure diffusion decreases only slowly as the soil surface is approached. Because of this, pressure diffusion accounts for more than $70 \%$ of the TKE source in the lowest one-third of the canopy, although all terms are small in these levels. Thus, our calculations support the hypothesis of Holland (1989) and the evidence of Shaw and Zhang (1992) that turbulence in the lowest levels of a canopy is largely induced by pressure perturbations.

Above $z=1.3 h$ or so, we observe that turbulent and pressure diffusion are opposed to each other, and that both reverse sign at about twice canopy height. 


\section{Turbulent Transport}

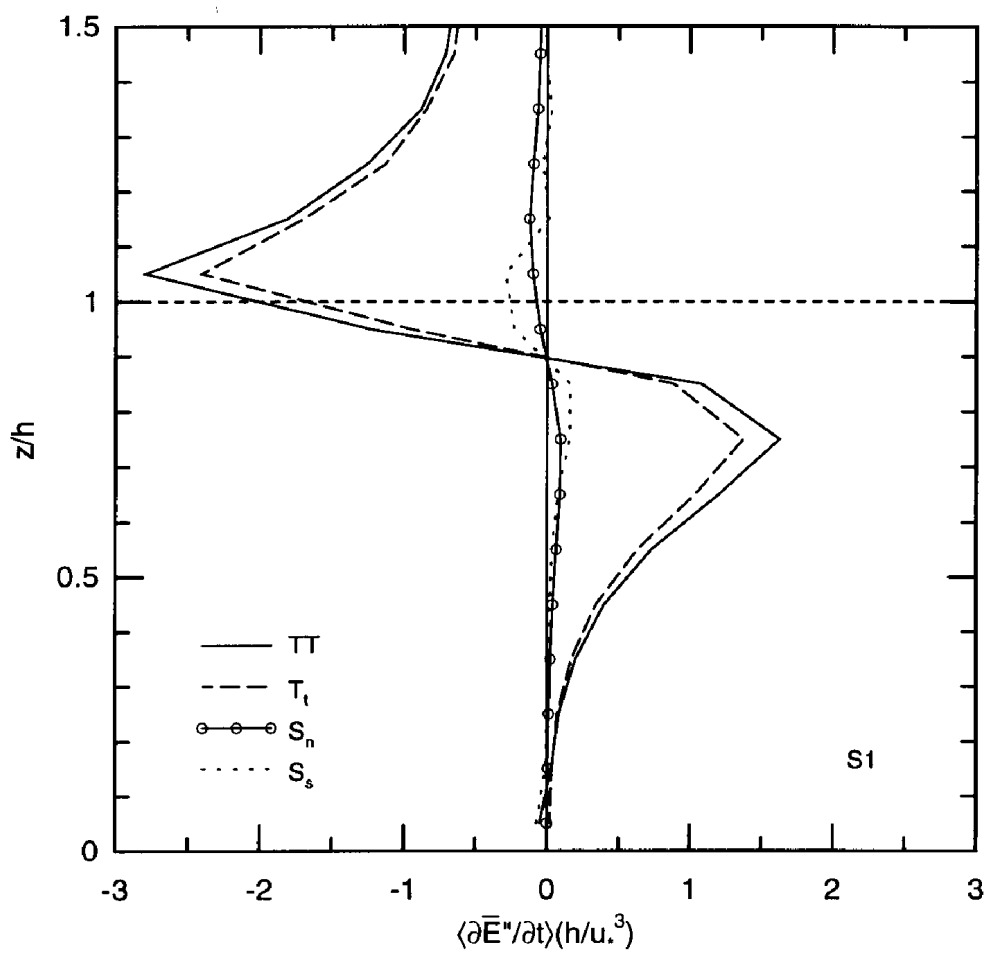

Figure 3. Normalized components of the turbulent transport term $(T T)$ for simulation $\mathrm{S} 1 . T_{t}$ is the resolved-scale component. $S_{n}$ and $S_{s}$ represent the normal and shear components of subgrid-scale diffusion of resolved-scale TKE $\left(T_{s g s}\right)$ respectively.

Pressure diffusion remains the smaller of the two, approximately $30 \%$ of the former, where they are not negligibly small. While the restrictive upper boundary condition might confuse the issue, this result does support surface-layer observations (McBean and Elliott, 1975) that turbulent and pressure diffusion are of opposite sign. Largeeddy simulations of the planetary boundary layer (Deardorff, 1980; Mason, 1989; Moeng and Wyngaard, 1989) have also shown the terms to be generally of reverse sign. As we have shown, though, this relationship does not extend to near-canopy and within-canopy levels, and the estimates of pressure diffusion attempted by Brunet et al. (1994), using parameterizations suggested by planetary boundary layer studies, produce results that are almost the exact opposite of our findings.

The influence of increased canopy density is shown in Figure 4 from simulation $\mathrm{S} 5$, which differs from simulation S1 only in terms of LAI (5 instead of 2). The main features of the budget remain (buoyancy is still insignificant in this second near-neutral case) but we note that shear production and canopy drag peak with values somewhat larger than for the less dense canopy, and that all terms, with the 


\section{Normalized TKE Budget}

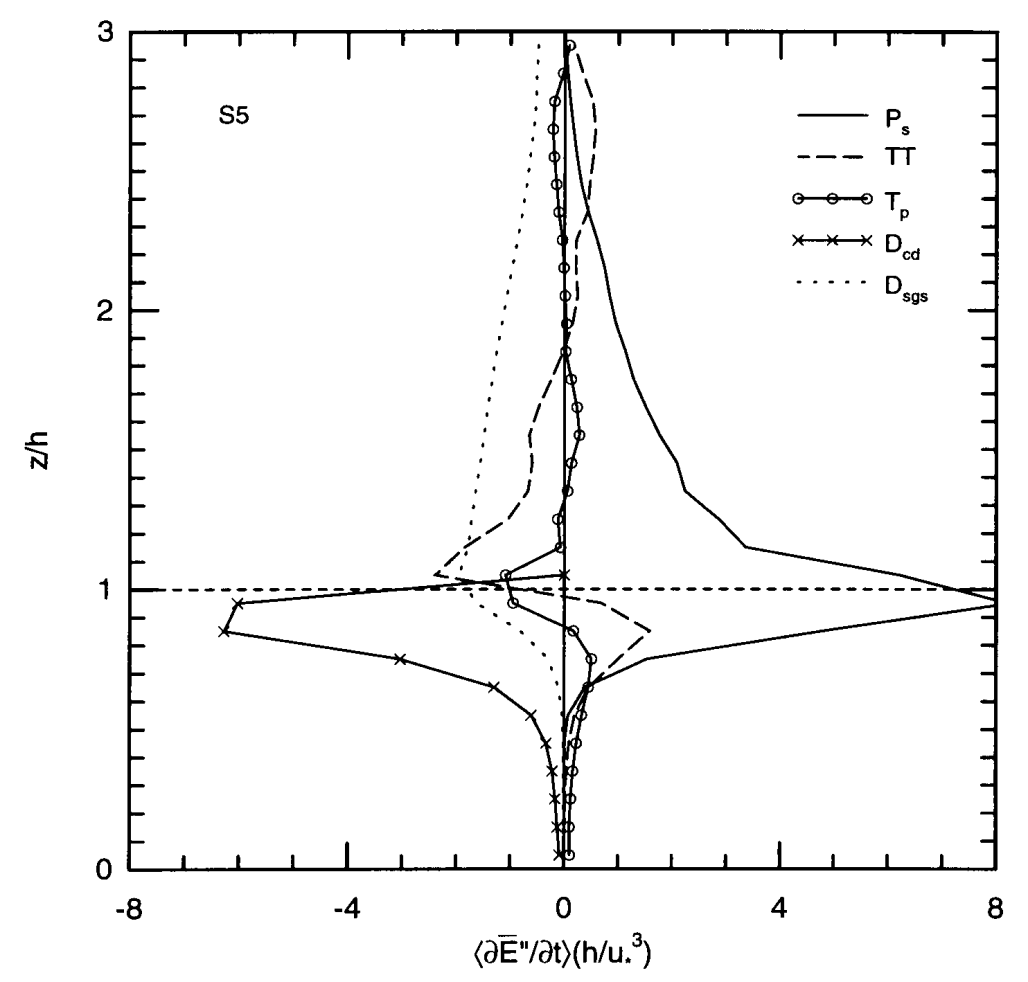

Figure 4. Normalized resolved-scale TKE budget for simulation $\mathrm{S} 5, h / L=-0.017$. Plot labels refer to budget terms in Equation (12), where $T T$ refers to the sum of $T_{t}$ and $T_{s g s}$.

exception of pressure diffusion, are extinguished more rapidly with depth in the forest.

The fact that the budget terms are expressed in normalized form must be kept in mind when evaluating differences between the two cases. Also to be considered is the fact that the mass flow through the domain was kept the same, so that, while the denser canopy experienced lower velocities, wind speeds in the upper part of the domain were higher. Given these factors, relative to the ratio $u_{*} / h$, the canopy with higher $L A I$ experienced stronger wind shear near the canopy top, creating a stronger rate of shear production. The more rapid decrease of shear production with depth is undoubtedly a consequence of the faster depletion of both the Reynolds stress and the wind shear within the denser canopy. Shear production decreases below $10 \%$ of its peak value at a somewhat higher level $(z / h \approx 0.65)$ than in the sparse canopy $(z / h \approx 0.55)$. Meyers and Baldocchi (1991) also found that normalized shear production decreased rapidly inside a deciduous forest with a leaf area index of 5 and their measurements showed shear production to be negligible below $z / h$ 
$\approx 0.85$. Again, the difference is likely due to the canopy architecture because, in their case, the foliage was concentrated more in the upper levels of the crown.

Canopy drag is larger near the top of the denser forest, as a direct result of the increased foliage density, but smaller (than the $L A I=2$ case) below, because of a more rapid diminishment of wind speed with depth. Decreases in both resolvedscale and subgrid-scale turbulence intensities deep within the denser canopy result in more rapid decreases in terms $T T$ and $D_{\text {sgs }}$. The latter is consistent with Shaw and Seginer (1985) who found dissipation to be negligible within a dense corn canopy $(L A I \approx 4.0$ ), while dissipation accounted for one-third of the loss in a sparse artificial wind tunnel canopy $(L A I \approx 0.5)$. Pressure diffusion, which is not strongly influenced by increased canopy density, is now the most important source of turbulence in the lower two-thirds of the canopy. In explanation, we suggest that pressure perturbations in the trunk space are largely the outcome of turbulence near tree-top height (Shaw et al., 1990) and are, thus, less strongly influenced by the flow reduction within the canopy that results from a denser canopy, than are the other terms.

A third example (Figure 5, simulation S8) includes the effect of strong heat flux and a smaller total flow rate, such that $h / L=-1.252$ representing strong instability. The leaf area index is 5 as in the previous case, S5. The additional production term is quite apparent in the upper canopy and the layers above. The buoyancy term decreases linearly to zero at the domain top because of the restrictive upper boundary condition, which dictates that the heat flux vanishes at $z / h=3$. In this strongly unstable case, buoyant production at tree-top level is about one-third as large as peak shear production. Normalized shear production is, itself, smaller by about 30\% than in the near neutral case S5, because convective mixing has reduced the wind shear near the canopy top relative to the magnitude of $u_{*} / h$. Leclerc et al. (1990) show equivalent influences of buoyancy on shear production.

Buoyancy decreases to zero by mid-canopy and is small but negative in the lower half. This is a direct consequence of the manner by which the heat source was specified, with source strength greatest in the upper canopy where the large leaf area density is exposed to high incoming radiation. With a non-conducting upper boundary, the simulation does not achieve thermal equilibrium and a small part of the heat from the upper leaves is directed downwards to warm the lowest air layers. This imposes weak stability on the bottom part of the canopy. While created here somewhat artificially, such a thermal structure has been reported as typical of daytime conditions (Kaimal and Finnigan, 1994). It might be pointed out that it is inappropriate to attempt to represent the stability of this sub-canopy situation by a parameter such as the flux Richardson number, which takes the ratio of the buoyancy term to the rate of shear production. As seen in Figure 5, at withincanopy levels pressure transport is the primary factor governing turbulence levels and shear production is insignificant, which is distinctly different from 'smooth wall' or above-canopy turbulent flows, where the flux Richardson number would be more appropriate. 


\section{Normalized TKE Budget}

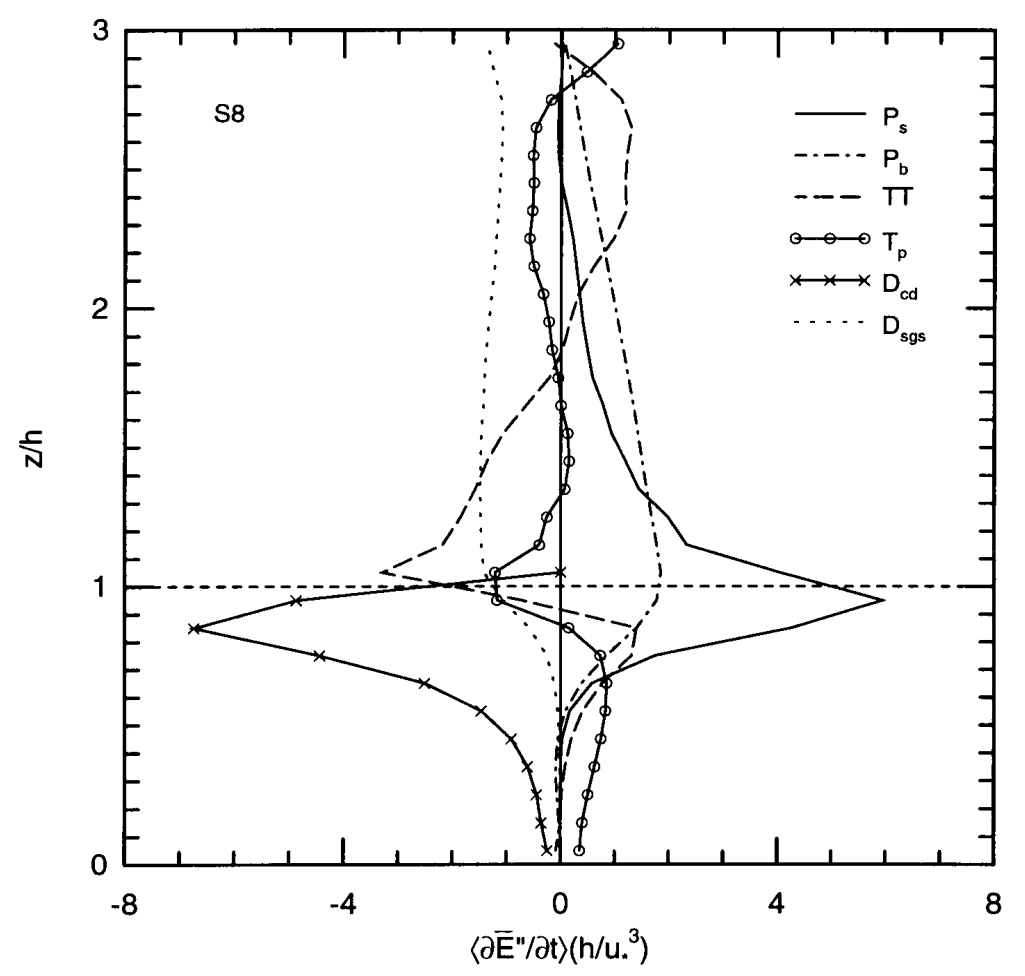

Figure 5. Normalized resolved-scale TKE budget for simulation $\mathrm{S} 8, h / L=-1.252$. Plot labels refer to budget terms in Equation (12), where $T T$ refers to the sum of $T_{t}$ and $T_{s g s}$.

Thermal instability appears to have a significant effect on the diffusion terms. Turbulent diffusion $(T T)$ becomes slightly larger in magnitude in both the negative region above the canopy and the positive region in the upper one-third of the domain. The positive region within the canopy shifts downward slightly. As far as the canopy is concerned, however, the biggest change occurs in the pressure diffusion term, which is substantially larger in the buoyant case (S8). Differences between the four cases with a leaf area index of 5 are shown more directly in Figure 6 for turbulent diffusion $(T T)$ and in Figure 7 for pressure diffusion $\left(T_{p}\right)$. For both terms, normalized profiles are influenced by the stability parameter $h / L$, rather than by the magnitude of the heat flux $Q_{*}$ (see Table I). The three near-neutral cases (S5, S6 and S7) are not ordered exactly by $h / L$ but differences are small and their profiles are almost coincident.

Stronger vertical motions created by thermal convection in case S8 induce larger pressure perturbations that impart greater amounts of turbulent kinetic energy in the lower canopy. In the lowest two-thirds of the depth of the canopy, pressure diffusion 


\section{Turbulent Transport}

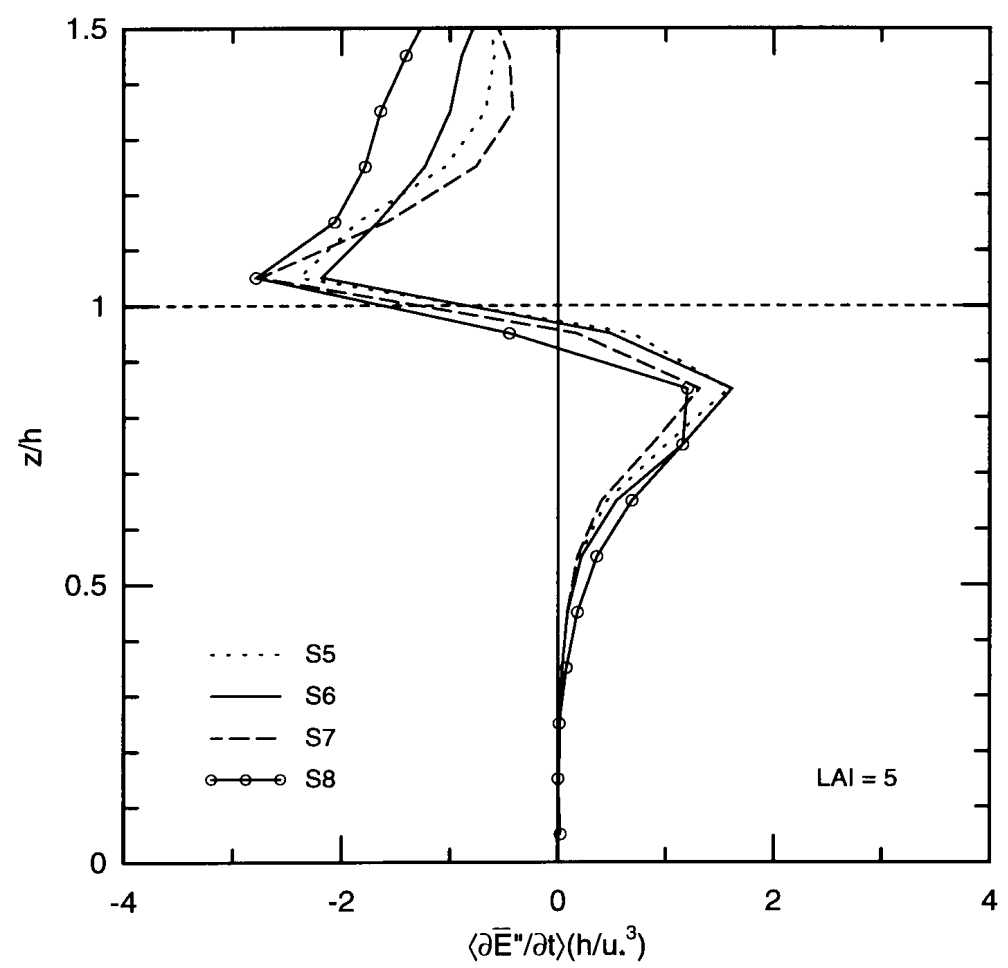

Figure 6. Normalized turbulent transport $(T T)$ for a dense canopy $(L A I=5)$. Plot labels are ordered in terms of increasingly negative $h / L$; S5 ( $h / L=-0.017)$, S6 (-0.064), S7 (-0.153), S8 (-1.252).

remains strong, while all other terms on the positive side fall off in exponential fashion (Figure 5). Thus, turbulence deep in the canopy is represented primarily by a balance between pressure diffusion and canopy drag. While the same can be claimed for the near-neutral cases (Figures 2 and 4), this result is most clearly demonstrated in the buoyant case.

\section{Summary and Conclusions}

The output of a large-eddy simulation has been used to calculate all terms of the resolved-scale turbulent kinetic energy budget for airflow within and above a forest canopy in which the computational domain extends vertically to three times the canopy height and to a little under ten times the canopy height in the horizontal directions. The TKE budget includes the traditional terms that represent shear and buoyant production, turbulent transport, and pressure transport; however, differences arise because the budget is for the resolved-scales of a large-eddy 


\section{Pressure Transport}

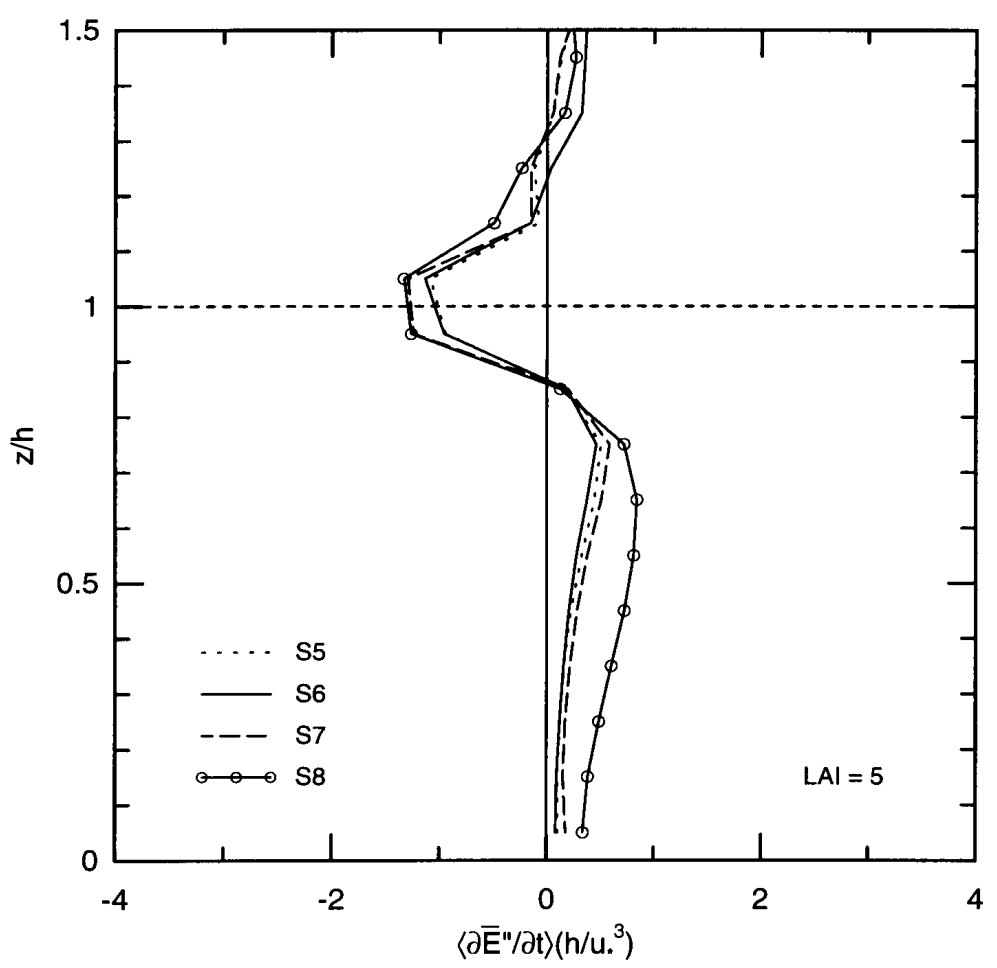

Figure 7. Normalized pressure transport $\left(T_{p}\right)$ for a dense canopy $(L A I=5)$. Plot labels are ordered in terms of increasingly negative $h / L ; \mathrm{S} 5(h / L=-0.017), \mathrm{S} 6(-0.064), \mathrm{S} 7(-0.153), \mathrm{S} 8(-1.252)$.

simulation. These scales are mathematically defined by a numerical filter that separates resolved-scale from subgrid-scale motions and sets a lower limit to the size of the resolvable eddies. As a consequence, a wake production term, which is sometimes found in a plant canopy budget, does not appear in the resolved-scale budget presented here. This is a result of our assumption that wake-scale turbulence is not resolved by the grid network. On the other hand, our budget does contain a term that represents the direct extraction of turbulent energy by plant element drag, which acts to suppress resolved-scale turbulent as well as mean flow kinetic energy. In addition, viscous dissipation effects are replaced here by a transfer of energy from the resolved to the subgrid-scales using a parameterized SGS diffusivity, although the descriptor 'dissipation' is retained.

Several simulations were run using different environmental forcing and plant canopy specifications. The plant canopy was defined as either sparse $(L A I=2)$ or dense $(L A I=5)$, and the effects of canopy heating on the surrounding air were specified by applying realistic profiles of solar heating at high and low levels. 
Thermal stability was defined by the ratio $h / L$, where $h$ is tree-top height and $L$ is the Obukhov length (calculated at the same level).

The effects of canopy density changes were seen in all profiles. The simulated profiles showed the normalized peak value of each budget term to be larger in the dense canopy than in the sparser stand. For example, normalized shear production for the dense canopy peaked at values approximately $30 \%$ larger than for the sparse canopy simulations. This feature was also observed in the normalized profiles of buoyant production, canopy drag, dissipation, and the transport terms. Accompanying this change, the magnitudes of the budget terms attenuated much more rapidly in the dense than in the more sparse canopy, indicating that, as expected, turbulent activity is suppressed in the lower portions of a dense forest when compared to a more open forest.

A significant aspect of this study is that the three-dimensional pressure perturbation field was numerically calculated, allowing the calculation of the pressure diffusion term throughout the domain, such that the effects of pressure diffusion could be compared to the other budget terms. As far as canopy TKE budgets are concerned, other than studies where estimates were based on budget residuals, we believe this to be the first time that such a comparison has been presented. The general features of the budget are that, (i) well above the canopy, there is an approximate balance between production and conversion to subgrid-scale motion (dissipation as far as resolved-scale motions are concerned); (ii) in this same region, turbulent and pressure transport roughly oppose one another but pressure diffusion is only about $30 \%$ of the magnitude of turbulent diffusion; (iii) at the canopy top, production is balanced by plant canopy drag effects, the transfer of energy to the subgrid-scales (dissipation), and by both transport mechanisms, where turbulent and pressure transport counteracted over one half of the production; (iv) in the lowest two-thirds of the canopy, production and subgrid-scale transfer become negligible, and the primary balance is between the loss due to canopy drag effects and the gain due to transport. Further, pressure transport is the dominant source in the lowest levels of the forest. In our simulations, pressure transport accounts for more than $70 \%$ of the TKE source in the lowest one-third of the sparse canopy, and is the most important source term in the lowest two-thirds of the dense canopy, especially under convective conditions.

These findings must be tempered with the understanding that they are the outcome of a numerical simulation and not direct field observation. The most important limitations of our model concern the relatively small size of the domain and the imposed boundary conditions, particularly that of the rigid but frictionless upper lid. Nevertheless, we believe that our simulations provide insight into canopy dynamics not previously available. 


\section{Acknowledgements}

The first author would like to thank the United States Air Force for his selection to attend University of California, Davis, under the AFIT/CI program. In addition, the authors would like to acknowledge Peter Sullivan and Chin-Hoh Moeng at the National Center for Atmospheric Research and Kyaw Tha Paw U at the University of California, Davis, for their generous contributions, both conceptual and numerical.

The computations presented in this manuscript were performed on a Cray YMP 8/864 courtesy of the National Center for Atmospheric Research. None of this work would have been possible without the financial support provided by the National Science Foundation under grant Nos. ATM-92-16345 and ATM-95-21586.

\section{References}

Andren, A., Brown, A. R., Graf, J., Mason, P. J., Moeng, C.-H., Nieuwstadt, F. T. M., and Schumann, U.: 1994, 'Large-Eddy Simulation of a Neutrally Stratified Boundary Layer: A Comparison of Four Computer Codes', Quart. J. Roy. Meteorol. Soc. 120, 1457-1484.

Brunet, Y., Finnigan, J. J., and Raupach, M. R.: 1994, 'A Wind Tunnel Study of Air Flow in Waving Wheat: Single Point Velocity Measurements', Boundary-Layer Meteorol. 70, 95-132.

Deardorff, J. W.: 1980, 'Stratocumulus-Capped Mixed Layers Derived From a Three-Dimensional Model', Boundary-Layer Meteorol. 18, 495-527.

Fox, D. G. and Orzag, S. A.: 1973, 'Pseudospectral Approximation to Two-Dimensional Turbulence', J. Comput. Phys. 11, 612-619.

Holland, J. Z.: 1989, 'On Pressure-Driven Wind in Deep Forests', J. Appl. Meteorol. 28, 1349-1355.

Kaimal, J. C. and Finnigan, J. J.: 1994, Atmospheric Boundary Layer Flows: Their Structure and Measurement, Oxford University Press, New York, 289 pp.

Kanda, M. and Hino, M.: 1994, 'Organized Structures in Developing Turbulent Flow Within and Above a Plant Canopy, Using a Large Eddy Simulation', Boundary-Layer Meteorol. 68, 237257.

Leclerc, M. Y., Beissner, K. C., Shaw, R. H., den Hartog, G., and Neumann, H. H.: 1990, 'The Influence of Atmospheric Stability on the Budgets of the Reynolds Stress and Turbulent Kinetic Energy Within and Above a Deciduous Forest', J. Appl. Meteorol. 29, 916-933.

Lesnik, G. E.: 1974, 'Results of Measurement of Turbulent Energy Balance Components in a Layer of Vegetation', Izv. Atmos. Oceanic Phys. 10, 652-655.

Maitani, T. and Seo, T.: 1985, 'Estimates of Velocity-Pressure and Velocity-Pressure Gradient Interactions in the Surface Layer Over Plant Canopies', Boundary-Layer Meteorol. 33, 51-60.

Mason, P. J.: 1989, 'Large-Eddy Simulation of the Convective Boundary Layer', J. Atmos. Sci. 46, 1492-1516.

Mason, P. J.: 1994, 'Large-Eddy Simulation: A Critical Review of the Technique', Quart. J. Roy. Meteorol. Soc. 120, 1-26.

McBean, G. A. and Elliott, J. A.: 1975, 'The Vertical Transports of Kinetic Energy and Pressure in the Boundary Layer', J. Atmos. Sci. 32, 753-766.

Meyers, T. P. and Baldocchi, D. D.: 1991, 'The Budgets of Turbulent Kinetic Energy and Reynolds Stress Within and Above a Deciduous Forest', Agric. For. Meteorol. 53, 207-222.

Meyers, T. P. and Paw U, K. T.: 1986, 'Testing of a Higher-Order Closure Model for Flow Within and Above Plant Canopies', Boundary-Layer Meteorol. 37, 297-311.

Meyers, T. P. and Paw U, K. T.: 1987, 'Modeling the Plant Canopy Micrometeorology with HigherOrder Closure Principles', Agric. For. Meteorol. 41, 143-163.

Moeng, C.-H.: 1984, 'A Large-Eddy Simulation Model for the Study of Planetary Boundary-Layer Turbulence', J. Atmos. Sci. 41, 2052-2062. 
Moeng, C.-H. and Wyngaard, J. C.: 1988, 'Spectral Analysis of Large-Eddy Simulations of the Convective Boundary Layer', J. Atmos. Sci. 45, 3573-3587.

Moeng, C.-H. and Wyngaard, J. C.: 1989, 'Evaluation of Turbulent Transport and Dissipation Closures in Second-Order Modeling', J. Atmos. Sci. 46, 2311-2333.

Nieuwstadt, F. T. M., Mason, P. J., Moeng, C.-H., and Schumann, U.: 1993, 'Large-eddy Simulation of the Convective Boundary Layer: A Comparison of Four Computer Codes', in F. Durst, R. Friedrich, B. E. Launder, F. W. Schmidt, U. Schumann and J. H. Whitelaw (eds.), Turbulent Shear Flows 8, Springer-Verlag, Berlin, pp. 343-367.

Patton, E. G., Shaw, R. H., Paw U, K. T., and Moeng, C.-H.: 1994, 'A Comparison of Two Large-Eddy Simulations of Turbulent Flow Above and Within a Forest Canopy', in Proc. Twenty-First Conf. of the Amer. Meteorol. Soc. on Agricultural and Forest Meteorol., San Diego, California, pp. 88-91.

Raupach, M. R., Coppin, P. A., and Legg, B. J.: 1986, 'Experiments on Scalar Dispersion Within a Model Plant Canopy. Part I: The Turbulence Structure', Boundary-Layer Meteorol. 35, 21-52.

Raupach, M. R. and Shaw, R. H.: 1982, 'Averaging Procedures for Flow Within Vegetation Canopies', Boundary-Layer Meteorol. 22, 79-90.

Shaw, R. H., den Hartog, G., and Neumann, H. H.: 1988, 'Influence of Foliar Density and Thermal Stability on Profiles of Reynolds Stress and Turbulence Intensity in a Deciduous Forest', Boundary-Layer Meteorol. 45, 391-409.

Shaw, R. H., Paw U, K. T., Zhang, X. J., Gao, W., den Hartog, G., and Neumann, H. H.: 1990, 'Retrieval of Turbulent Pressure Fluctuations at the Ground Surface Beneath a Forest', Boundary-Layer Meteorol. 50, 319-338.

Shaw, R. H. and Schumann, U.: 1992, 'Large-Eddy Simulation of Turbulent Flow Above and Within a Forest', Boundary-Layer Meteorol. 61, 47-64.

Shaw, R. H. and Seginer, I.: 1985, 'The Dissipation of Turbulence in Plant Canopies', in 7th Symp. of the Amer. Meteorol. Soc. on Turbulence and Diffusion, Boulder, Colorado, pp. 200-203.

Shaw, R. H. and Zhang, X. J.: 1992, 'Evidence of Pressure-Forced Flow in a Forest', Boundary-Layer Meteorol. 58, 47-64.

Sigmon, J. T., Knoerr, K. R., and Shaughnessy, E. J.: 1983, 'Microscale Pressure Fluctuations in a Mature Deciduous Forest', Boundary-Layer Meteorol. 27, 345-358.

Wilson, J. D.: 1988, 'A Second-Order Closure Model for Flow Through Vegetation', Boundary-Layer Meteorol. 42, 371-392.

Wilson, N. R. and Shaw, R. H.: 1977, 'A Higher Order Closure Model for Canopy Flow', J. Appl. Meteorol. 16, 1197-1205.

Zhuang, Y. and Amiro, B. D.: 1994, 'Pressure Fluctuations During Coherent Motions and Their Effects on the Budgets of Turbulent Kinetic Energy and Momentum Flux Within a Forest Canopy', $J$. Appl. Meteorol. 33, 704-711. 